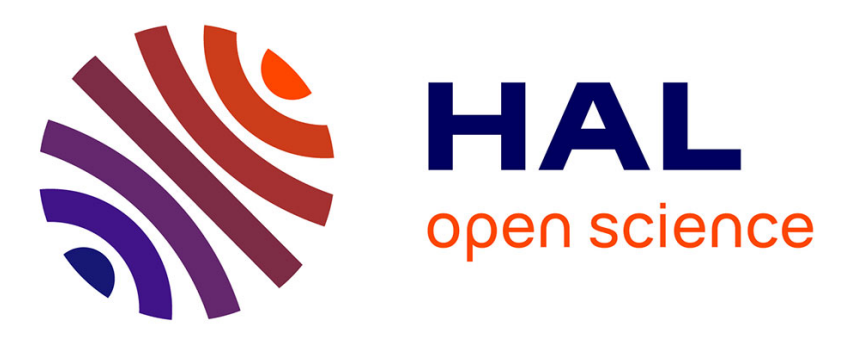

\title{
Acquired biodegradability of polyethylenes containing pro-oxidant additives
}

Marek Koutny, Martine Sancelme, Catherine Dabin, Nicolas Pichon, A.M.

Delort, Jacques Lemaire

\section{- To cite this version:}

Marek Koutny, Martine Sancelme, Catherine Dabin, Nicolas Pichon, A.M. Delort, et al.. Acquired biodegradability of polyethylenes containing pro-oxidant additives. Polymer Degradation and Stability, 2006, 91, pp.1495-1503. 10.1016/j.polymdegradstab.2005.10.007 . hal-00021890

\section{HAL Id: hal-00021890 \\ https://hal.science/hal-00021890}

Submitted on 27 Feb 2007

HAL is a multi-disciplinary open access archive for the deposit and dissemination of scientific research documents, whether they are published or not. The documents may come from teaching and research institutions in France or abroad, or from public or private research centers.
L'archive ouverte pluridisciplinaire HAL, est destinée au dépôt et à la diffusion de documents scientifiques de niveau recherche, publiés ou non, émanant des établissements d'enseignement et de recherche français ou étrangers, des laboratoires publics ou privés. 


\title{
Acquired biodegradability of polyethylenes containing pro-oxidant additives
}

\author{
Marek Koutny ${ }^{\text {a,b,c }}$, Martine Sancelme ${ }^{\mathrm{b}}$, Catherine Dabin ${ }^{\mathrm{a}}$, Nicolas Pichon ${ }^{\mathrm{a}}$, \\ Anne-Marie Delort ${ }^{\mathrm{b}, *}$, Jacques Lemaire ${ }^{\mathrm{a}}$ \\ ${ }^{a}$ Centre National d Evaluation de Photoprotection (CNEP), Universite Blaise Pascal, 63177 Aubiere Cedex, France \\ ${ }^{\mathrm{b}}$ Laboratoire de Synthese Et Etude de Systemes a Interet Biologique (SEESIB), UMR 6504 CNRS, Ensemble Universitaire des Cezeaux, \\ Univer site Blaise Pascal, 63177 Aubiere Cedex, France \\ ${ }^{c}$ Tomas Bata University in Zlin, Faculty of Technology, Department of Environmental Engineering, 76272 Zlín, Czech Republic
}

Received 6 September 2005; received in revised form 3 October 2005; accepted 7 October 2005

Available online 13 December 2005

\begin{abstract}
Biodegrability of high density polyethylene film (HDPE) and low density polyethylene film (LDPE) both containing a balance of antioxidants and pro-oxidants was studied with defined microbial strains particularly with Rhodococcus rhodochrous and Nocardia asteroides in mineral medium. After an abiotic pre-treatment consisting of photooxidation and thermo-oxidation corresponding to about 3 years of outdoor weathering the samples were inoculated, incubated up to 200 days and during the period their metabolic activities were followed by measuring adenosine triphosphate content. Simultaneously the cultures were also monitored by optical microscopy and FTIR spectroscopy. The first initial phase of fast growth caused by the presence of low molecular extractable compounds was followed by a long period of stabilized metabolic activity suggesting that microorganisms continued to gain energy from the substrate but evidently at a much slower rate. Complementary analysis performed at the end of incubation revealed that during the experiment time biodegradation processes probably affected surface layer of materials only. (c) 2005 Elsevier Ltd. All rights reserved.
\end{abstract}

Keywords: Adenosine triphosphate; Polyethylene; B iodegradation; Pro-oxidant; Photooxidation; Thermo-oxidation

\section{Introduction}

Traditionally the biodegradability of pro-oxidant activated PE has been evaluated in complex media like soil, waste water sludge or compost. The approach brings several advantages like availability of very diverse microbial inoculum or close relation to the real conditions in the nature and/or in waste treatment processes. Recently two studies presented quantitative indications of the biodegradability of PE film containing a pro-oxidant additive. Chiellini et al. [1] followed incubation of previously thermally exposed film in soil or in compost conditions and reported $50-60 \%$ and $80 \%$ mineralization, respectively, after approximately one and a half year of incubation.

\footnotetext{
* Corresponding author. Tel.: +3347340 77 14; fax: +33473407717.
} E-mail address: A-Marie.DELORT@univ-bpclermont.fr (A.-M. Delort).
Jakubowicz [2] claims even $60 \%$ mineralization during only 6-month incubation.

Although such experiments are relevant to natural conditions, the chemical and biological complexities of the system make it difficult to gain a better insight into detailed mechanisms of biodegradation. For a better understanding of the process fundamentals the key task is to define more controlled experimental conditions i.e. experiments with identified microbial strains in a medium formulated from defined chemical compounds. Establishing such controlled conditions was the key effort throughout this study; this approach appeared to be complementary to previous works using complex media.

During previous experiments $[3,4]$ bacterial and fungal strains promising for oxidized PE degradation were selected and it was shown that at least some of them form dense biofilm. Scanning electron microscopy also revealed signs of 
bioerosion on the material surface. Some of the strains were utilized also in the present work.

To follow growth and development of microbial cultures the method of determination of adenosine triphosphate (ATP) and adenosine diphosphate (ADP) was adopted. ATP is the key molecule of all living cell energetic metabolism. Its level reflects metabolic activity of a culture. Moreover the adenylates can be extracted and determined from entire sample, which means simultaneously from both the biofilm on the material surface and the surrounding liquid medium, the assay is extremely sensitive and the response linear across several orders of magnitude.

\section{Materials and methods}

\subsection{Tested material}

The material samples were transparent HDPE film $20 \mu \mathrm{m}$ thick and transparent LDPE film $60 \mu \mathrm{m}$ thick. Both films contained iron photo-inducer, different from the Scott/Gilead compound (Schulman - Bornen, Belgium) supplying radicals through a photo-redox process and an organometallic type thermo-inducer (EPI, Vancouver, Canada) catalysing the primary hydroperoxide decomposition. Both additives were present in both films. To balance the prodegradant activity of the photo- and thermo-inducer during the first year of storage and use under indoor conditions phenolic antioxidants were used in the blends. Due to the added antioxidants the thermalinduction period was longer than $400 \mathrm{~h}$ at $60^{\circ} \mathrm{C}$ in dark.

\subsection{Abiotic treatment}

The samples were exposed for $120 \mathrm{~h}$ in the accelerated photoageing chamber SEPAP $12.24[3,5](\lambda \geq 300 \mathrm{~nm}$, temperature of the exposed surface was set at $60 \pm 1{ }^{\circ} \mathrm{C}$ ). After the exposure in SEPAP the samples were considered sterile and treated in a sterile way. The first $20 \mathrm{~h}$ of exposure corresponded to the photochemical induction period (total photo-transformation of phenolic antioxidants into inactive compounds). During the additional $100 \mathrm{~h}$ fast photooxidation proceeded and at the end of the period the absorbance measured at $1715 \mathrm{~cm}^{-1}$ should be equal or higher to $x / 100$, where $x$ was the film thickness in microns. At this level of oxidation spontaneous fragmentation of the film should be observed. For the examined PE films $100 \mathrm{~h}$ of exposure in SEPAP 12.24 corresponds to 3 months weathering under European outdoor conditions in the period from March to October.

Exposure in SEPAP was followed by $300 \mathrm{~h}$ of exposure in an aerated oven at $60{ }^{\circ} \mathrm{C}$. Based on the activation energy determined when thermo-oxidation proceeded without restraint (after the total consumption of phenolic antioxidants) $300 \mathrm{~h}$ of thermo-oxidation at $60{ }^{\circ} \mathrm{C}$ was equivalent to $2-3$ years of thermo-oxidation at room temperature in the dark (particles buried in the soil or particles not exposed to sunlight).

It should be emphasized that instead of exposure in SEPAP 12.24 under dry conditions the abiotic pre-treatment also included $120 \mathrm{~h}$ exposure in SEPAP $12.24 \mathrm{H}$ where the films were exposed submerged under $2 \mathrm{~mm}$ water layer with the controlled temperature $\left(60 \pm 1{ }^{\circ} \mathrm{C}\right)$ where the constant concentration of dissolved oxygen is assured by re-oxygenation in the external circuit. The UV light $(\lambda \geq 300 \mathrm{~nm})$ passes through the thin water film without any intensity decrease. The experiment was designed to check if release of any water extractable compounds from the material tested could influence its photo-oxidisability and thermo-oxidisability in the surface layer in the aqueous environment.

To obtain more uniform and more convenient particle size the exposed film, already highly fragmented and very fragile was passed through a metallic screen with hole diameters of about $1 \mathrm{~mm}$.

In the actual phase of thermo-oxidation, the apparent activation energy was determined to be $100 \mathrm{~kJ} / \mathrm{mol}$, whereas in the phase corresponding to the thermal-induction period during which phenolic antioxidant counteracted the influence of the pro-oxidant additive, the apparent activation energy was only $40 \mathrm{~kJ} / \mathrm{mol}$.

\subsection{Strains of bacteria and fungi}

Rhodococcus rhodochrous ATC 29672, Aspergillus flavus ATCC 26873, Mortierella alpina ATCC 36965, Cladosporium cladosporoides ATCC 20251 were purchased from American Type Culture Collection; Nocardia asteroides LAB 911 was previously isolated in our laboratory [4].

\subsection{Medium and conditions of the cultivation}

The mineral medium utilized throughout the study had the following composition: $3.8 \mathrm{~g} \mathrm{Na}_{2} \mathrm{HPO}_{4} \cdot 12 \mathrm{H}_{2} \mathrm{O}, 1.8 \mathrm{~g}$ $\mathrm{KH}_{2} \mathrm{PO}_{4}, 0.02 \mathrm{~g} \mathrm{MgSO}_{4} \cdot 7 \mathrm{H}_{2} \mathrm{O}, 0.03 \mathrm{~g} \mathrm{Fe}\left(\mathrm{NH}_{4}\right)_{2}\left(\mathrm{SO}_{4}\right)_{2} \cdot 6 \mathrm{H}_{2} \mathrm{O}$, $0.01 \mathrm{~g} \mathrm{CaCl}_{2} \cdot 2 \mathrm{H}_{2} \mathrm{O}, 0.5 \mathrm{~g} \mathrm{NaCl}, 0.3 \mathrm{~g} \mathrm{NH}_{4} \mathrm{Cl}$ and $1 \mathrm{ml}$ of trace element solution per liter. The trace element solution contained $0.20 \mathrm{~g} \mathrm{MnSO}_{4}, 0.029 \mathrm{~g} \mathrm{H}_{3} \mathrm{BO}_{3}, 0.022 \mathrm{~g} \mathrm{ZnSO}_{4} \cdot 7 \mathrm{H}_{2} \mathrm{O}, 1.0 \mathrm{~g}$ $\mathrm{Na}_{2} \mathrm{MoO}_{4}$, traces of $\mathrm{Co}\left(\mathrm{NO}_{3}\right)_{2}$, and traces of $\mathrm{CuSO}_{4}$ dissolved in $500 \mathrm{ml}$ of water.

Thimerasol at final concentration $0.01 \%(\mathrm{w} / \mathrm{w})$ was added into abiotic control cultivations as a growth inhibitor.

In general incubations for microscopy, GPC and spectroscopy observation were done in closed $100 \mathrm{ml}$ glass flasks with $10 \mathrm{ml}$ of media, incubation for ATP level determination in $4 \mathrm{ml}$ closed glass vials with $0.4 \mathrm{ml}$ of media. In both cases the head-spaces were sufficiently large to provide the cultures with oxygen; moreover the flasks and vials were opened monthly so that the head-space air could be refreshed. Gastight sealing of the vessels was necessary to prevent water evaporation during the long incubation. The cultures were kept at $27{ }^{\circ} \mathrm{C}$ with gentle shaking. PE substrate concentrations were about $4 \mathrm{mg} / \mathrm{ml}$ for flask cultures and about $5 \mathrm{mg} / \mathrm{ml}$ for cultures in vials.

\subsection{Transmission and ATR-FTIR}

Small fragments whose section could be as small as $1000 \mu \mathrm{m}^{2}$ were analysed by micro-FTIR spectrophotometry, 
the combination of FTIR spectrophotometry and IR microscopy (NEXUS, Thermo Nicolet). The IR beam entering the spectrophotometer was focused on a predefined $1000 \mu \mathrm{m}^{2}$ zone of the small fragment and a micro-FTIR spectrum was recorded in the transmission mode with a resolution of $\pm 2 \mathrm{~cm}^{-1}$ and absorbance defined with \pm 0.001 precision. The optical path was determined using the main absorption band of HDPE or LDPE at 1468 and $1465 \mathrm{~cm}^{-1}$, respectively. $\mu$ ATR-FTIR spectra were recorded on a Nicolet 800 FTIR spectrometer connected to NIC-PLAN IR microscope.

\subsection{Size exclusion chromatography (SEC)}

The whole cultures were dried in vacuum, polymer was dissolved in a defined volume of added 1,3,5-trichlorobenzene at $160{ }^{\circ} \mathrm{C}$ to obtain $0.1 \%$ solution and filtered. Santonox $\mathrm{R}$ phenolic antioxidant of $250 \mathrm{ppm}$ was present in the solvent to prevent the oxidation of the sample by oxygen. Molecular weight distributions were determined with high temperature GPC system Waters 150C ALC/GPC. Solutions of polystyrene molecular weight standards were used for the calibration.

\subsection{ATP and ADP assays}

ATP Biomass Kit HS by Biothema (Sweden) was used for ATP determination. For each determination ATP from the entire culture in a $4 \mathrm{ml}$ vial was extracted. At least two vials were analysed for each time point. ADP was determined after transformation of ADP to ATP directly in the luminometer cuvette. Reaction mixture contained $30 \mu \mathrm{l}$ of the sample extract, $240 \mu \mathrm{l}$ of diluent B from the ATP kit, amended with $20 \mathrm{mM} \mathrm{KCl}$ and $2 \mathrm{mM} \mathrm{MgSO}_{4}$, and $10 \mu \mathrm{l}$ of solution containing $0.1 \mathrm{M}$ phosphoenolpyruvate and $5 \mathrm{mg} / \mathrm{ml}$ protein kinase in $0.05 \mathrm{M}$ Tris-acetate buffer $\mathrm{pH} 7.2$ [6]. The mixture was incubated for $45 \mathrm{~min}$ at $35^{\circ} \mathrm{C}$, equilibrated to the lab temperature for $15 \mathrm{~min}$ and then the light producing reaction was started by the addition of $60 \mu \mathrm{l}$ ATP reagent HS (Biothema) and reconstituted with $2.5 \mathrm{ml}$ of water. A blank experiment was done simultaneously to correct results for the background signal of the reagents.

\subsection{Microscopy techniques}

PE film fragments were separated from the suspension by carefully pipetting out the water phase. For the optical microscopy microorganisms on the PE film fragments were initially fixed with fixative solution containing formaldehyde and ruthenium red and then stained with safranine.

Samples for scanning electron microscopy were prepared with the procedure described in Ref. [4].

\subsection{NMR spectroscopy}

NMR spectra were recorded after filtration of the media through $0.2 \mu \mathrm{m}$ porosity filter.

NMR samples were prepared as follows: supernatants (540 $\mu \mathrm{l})$ produced from biodegradation tests were supplemented with $60 \mu \mathrm{l}$ of a $5 \mathrm{mM}$ solution of $\mathrm{TSPd}_{4}$ (sodium tetra deuterated trimethylsilyl propionate, Eurisotop) in $\mathrm{D}_{2} \mathrm{O}$ (Eurisotop). $\mathrm{D}_{2} \mathrm{O}$ was used for locking and shimming while $\mathrm{TSPd}_{4}$ constituted a reference for chemical shifts $(0 \mathrm{ppm})$ and quantification. ${ }^{1} \mathrm{H}$ NMR spectra were recorded at $500.13 \mathrm{MHz}$ on a Bruker Avance 500 spectrometer at $21{ }^{\circ} \mathrm{C}$ with $5 \mathrm{~mm}$-diameter tubes containing $600 \mu \mathrm{l}$ of sample. One hundred and twenty-eight scans were collected $\left(90^{\circ}\right.$ pulse, $4.67 \mathrm{~s}$ acquisition time, $3.0 \mathrm{~s}$ relaxation delay, $7002.80 \mathrm{~Hz} \mathrm{SW}, 65536$ data points). Water signal was eliminated by pre-saturation. No filter was applied before Fourier transformation but a baseline correction was performed on spectra before integration with Bruker software. Under these conditions, the limit of quantification is in the range of $0.05 \mathrm{mM}$.

\section{Results}

\subsection{Abiotic treatment}

The following procedure was aimed to prepare samples corresponding to the material after weathering during the predefined time period in outdoor conditions (see Section 2.2). After this period by the action of the pro-oxidants, light and heat the material should be substantially chemically transformed and thus more susceptible to a microbial attack.

To obtain samples required for the experiments with microorganisms large areas of HDPE or LDPE film were exposed in SEPAP 12.24 photoageing unit. The unit was originally designed for the exposure of small sized samples. In case of HDPE samples with larger surface some problems arose due to mechanical perturbation caused by the air circulation necessary to maintain the sample temperature at $60{ }^{\circ} \mathrm{C}$ and spontaneous fragmentation of the sample was observed prematurely i.e. before the oxidation extent corresponding to an absorption increase at $1712 \mathrm{~cm}^{-1}$ was equal to $x / 100$, where $x$ is the thickness of the film in microns (see Section 2.2). Indeed a PE film usually lost $50 \%$ of its mechanical properties when the oxidation extent corresponding to an absorbance increase at $1712 \mathrm{~cm}^{-1}$ equals to $x / 1000$, so it was not surprising that the air circulation could provoke cracking when the oxidation extent equaled only $0.4 x / 100$ after $70 \mathrm{~h}$ of exposure in SEPAP 12.24 .

The fragments of photo-oxidized film were transferred to a sterile box and submitted to thermo-oxidation at $60{ }^{\circ} \mathrm{C}$ during $528 \mathrm{~h}$. At the end of the abiotic treatment the absorbance increase at $1712 \mathrm{~cm}^{-1}$ determined by micro-FTIR spectroscopy was $3 x / 100$.

During the abiotic oxidation the molecular weight of the polymer decreased dramatically (Table 1). Surprisingly also narrowing of the molecular weight distribution curve was observed (Fig. 1).

As will be described in detail later, some substances can be released from the oxidized plastic to the water phase. Motivated by concern about whether the pro-oxidant additives could be washed out of the material in humid or aquatic environment an experiment was carried out where the samples were exposed submersed in water in the photoageing unit SEPAP 
Table 1

Average molecular weight of the polymer samples before and after the abiotic treatment (see Section 3.1)

\begin{tabular}{lrrl}
\hline Sample & \multicolumn{1}{l}{$M_{\mathrm{W}}$} & \multicolumn{1}{l}{$M_{\mathrm{N}}$} & $I_{\mathrm{P}}$ \\
\hline HDPE initial & 288000 & 34000 & 8.5 \\
HDPE after the abiotic treatment & 12800 & 5200 & 2.5 \\
LDPE initial & 24200 & 4800 & 5.0 \\
LDPE after the abiotic treatment & 16500 & 5300 & 3.1 \\
\hline
\end{tabular}

$M_{\mathrm{W}}$, weight average molecular weight; $M_{\mathrm{N}}$, number a verage molecular weight; $I_{\mathrm{P}}$, polydispersity index.

12.24 H. Our observation then proved that the course of the photooxidation was identical as for the dry conditions and thus the additives were not leached out in a significant extent.

\subsection{Metabolic activity of cells during incubation with the oxidized $P E$}

\subsubsection{Evolution of ATP content}

All of the following described experiments were done in medium containing only necessary growth supporting mineral ions and where the tested material was present as the only source of carbon and energy so that no objection could be raised that the microorganisms were profiting from other media component than the tested material.

Because in the previous studies $[3,4]$ the bacterial strains of $R$. rhodochrous and $N$. asteroides were found to be the most active in terms of biofilm formation on the PE surface, the most detailed attention was paid to the experiments with those microorganisms.
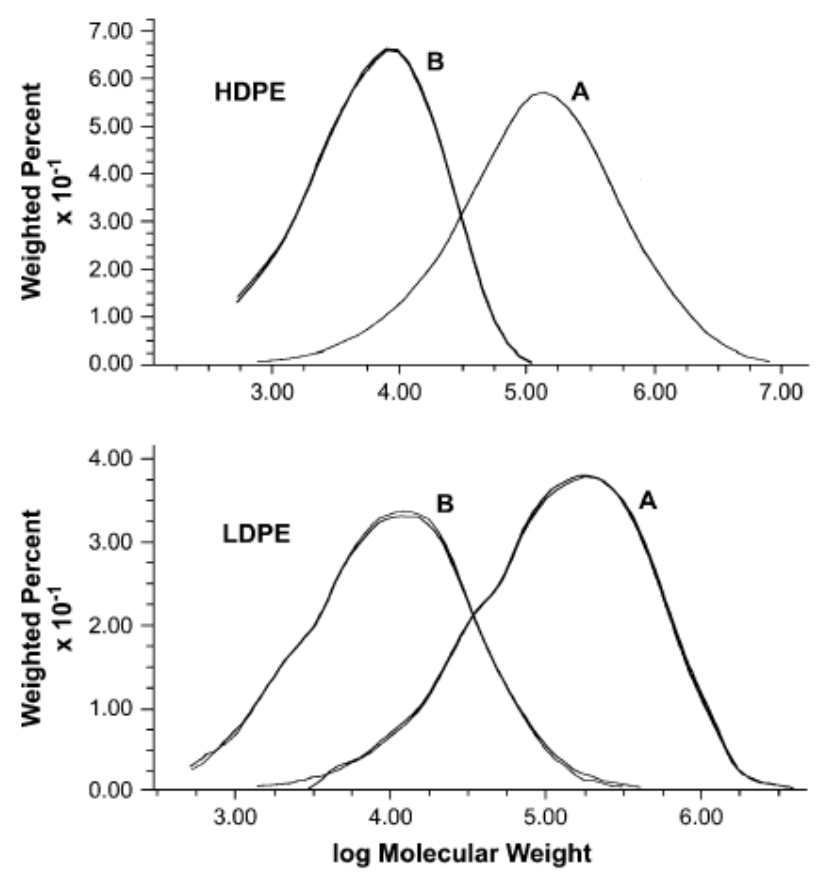

Fig. 1. Molecular weight distribution curves of the polyethylene films. (A) Initial material; (B) after the abiotic treatment (two curves of two parallel samples are displayed for each case).
With the aim of following the evolution of the microorganism culture and its metabolic activity, ATP content was determined in given time intervals. In the case of oxidized HDPE film and $R$. rhodochrous (Fig. 2) the initial phase after the contact with the oxidized PE film and the bacteria was characterized by a fast growth that peaked after about 3 or 4 days of incubation. The observed behaviour was provoked by the availability of the easily metabolisable, probably lower molecular weight substances, present in the treated PE film and/or extracted from the film to the aqueous medium. Then the ATP content dropped as a consequence of the above described "easy" substrate depletion. After approximately 50 days the ATP content, so the corresponding metabolic activity, stabilized itself and maintained virtually the same level up to the end of the observation i.e. up to more than 6 months. The situation was slightly different for the oxidized LDPE film. Here the initial growth was not so intense probably indicating a lower amount of substances being extracted from the material which could be eventually explained by the three times greater thickness of the film compared to HDPE and the corresponding smaller active surface. Nevertheless the ATP content remained at a level similar to the HDPE film.

To prove that the extracted matter really was responsible for the initial fast growth and to find out if the oxidized HDPE without water extractible substances still support bacterial growth another experiment was prepared. The sterile mineral medium and the oxidized HDPE film were combined in a ratio identical as for the previous incubation and shaken $24 \mathrm{~h}$ at $27^{\circ} \mathrm{C}$. At the end of this period the solid and the liquid were separated by filtration through a $0.2 \mu \mathrm{m}$ filter. The solid part was dried in vacuum at the laboratory temperature and cultures were prepared containing the same content of the material as described before for the oxidized HDPE or LDPE films. The liquid part was inoculated and $0.4 \mathrm{ml}$ volumes were distributed into vials. The metabolic activity in the cultures with the solid residue after the extraction peaked and then reached a steady level similar to the complete HDPE film. This suggested that the material still contained some part of the rapidly

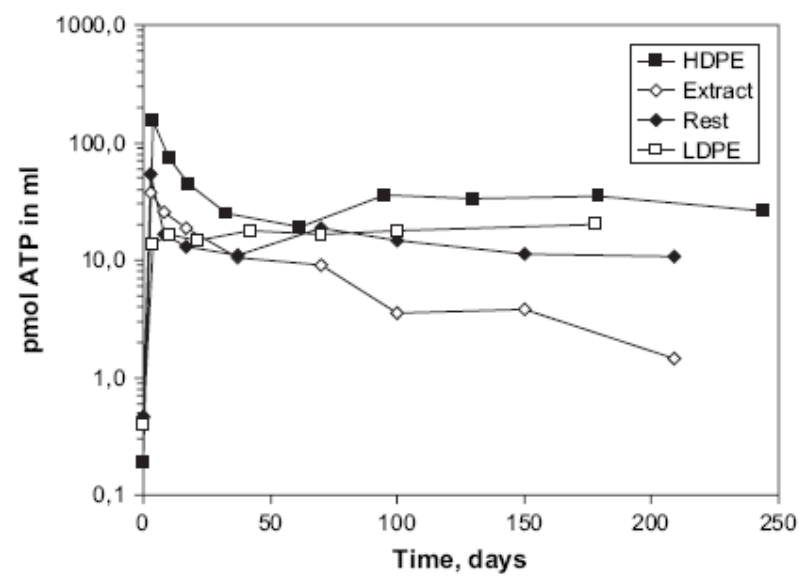

Fig. 2. Evolution of ATP content in $R$. rhodochrous cultures with oxidized PE films and control culture with the aqueous extract from the oxidized HDPE. 
metabolisable substances and after their consumption the culture stabilized its ATP content at a level not so far from the complete oxidized LPDE or HDPE films. In contrast to the above observations the cultures with the extract after the same initial growth and the consumption of extracted substances entered into a phase of a slow steady decline of the metabolic activity.

The ATP level curve of the extract could thus be used as some kind of reference demonstrating that the observed stable phase for the other curves was the consequence of a certain level of ongoing substrate supply from the oxidized polymer film to microorganisms.

In the case of $N$. asteroides culture after the initial growth the ATP level remained stable at a similar level for both oxidized HDPE and LDPE films (Fig. 3). Similarly, as in the case described above, the incubations with the HDPE film extract were also followed showing very slow but steady decrease in ATP level. It should be noted that the observation period was shorter than for $R$. rhodochrous.

\subsubsection{ADP/ATP ratio at the end of the cultivation}

The metabolic state of a microbial population can be better characterized with a knowledge of the other adenylate nucleotide contents. For the selected samples ADP contents were determined in addition to ATP. The ADP/ATP ratio can be considered as a measure of the cell's energetic state. To reach exponential growth cells must be rich in energy and that is why in this type of population most of the adenine nucleotide pool could be found in the form of ATP and the respective ADP/ATP ratio is low. The fact is well documented in the first line of Table 2 for the culture in rich complete Trypcase Soja medium at the end of the exponential growth phase (ADP/ ATP $=0.25$ ). Similar values could also be found for cultures with the oxidized HDPE and LDPE as the only substrate in mineral medium in the early phase of incubation (4 days).

Cells with a limited energetic supply cannot keep the ADP/ ATP ratio so low, hence all of the population cannot grow exponentially, but the cells are still alive with the working

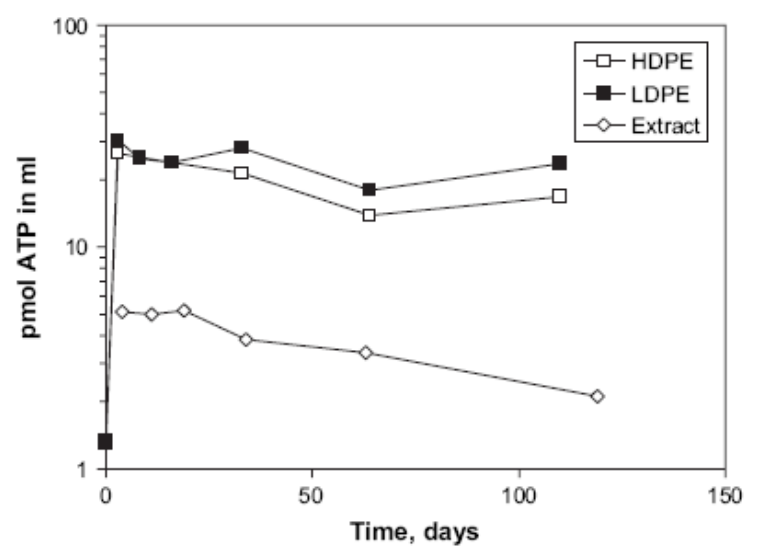

Fig. 3. Evolution of ATP content in $N$. asteroides cultures with oxidized PE films and control culture with the aqueous extract from the oxidized HDPE.
Table 2

ADP/ATP ratio for $R$. rhodochrous cultures during and at the end of the observation period

\begin{tabular}{lcr}
\hline Substrate & Incubation (days) & ADP/ATP \\
\hline TS medium & 4 & $0.25(0.02)$ \\
Oxidized HDPE & 4 & $0.14(0.03)$ \\
Oxidized LDPE & 4 & $0.43(0.09)$ \\
Oxidized HDPE & 250 & $2.2(0.21)$ \\
Oxidized LDPE & 185 & $2.2(0.31)$ \\
HDPE rest after the extraction & 200 & $2.5(0.01)$ \\
HDPE extract (control) & 200 & $6.0(0.11)$
\end{tabular}

TS medium, complete rich medium; standard deviations of the values are in brackets, $n \geq 2$.

metabolic machinery. It is also possible that in the bulk population some subpopulation of dividing cells still exist. The more the energy inputs are limited, the more the cells are starving and at some point they are no longer able to keep their metabolic functions and their structural integrity and enter the irreversible process of senescence. Those differences in cell energetic status are reflected also in the ADP/ATP ratio. For the cultures with the oxidized HDPE and LDPE films and the rest after the oxidized HDPE film extraction described earlier the values of the ratio were virtually in the same range (ADP/ATP about 2.2) whereas cultures with the extractable portion only gave clearly higher number documenting higher level of the energy deprivation (ADP/ATP about 6.0).

Similar analysis was done for the $N$. asteroides cultures (Table 3). Again the ADP/ATP values were lower for the cultures with tested materials than for the cultures with the same extract only but the difference was not so pronounced. The observation can be explained by shorter incubation period (120 days instead of 200 days).

The values obtained showed that the cell populations in the presence of the oxidized PE samples were in better energetic state than the control cultures with the extract only. It suggests that the cell after the initial period of exponential growth continued to extract substances from the oxidized PE and gained some energy by their metabolism. But it is also obvious that the values even lower than those of the control incubation were still rather high suggesting that the cells had only limited energetic supply and that the energy generation from the tested material is slow and difficult.

\subsection{Consumption of extractable molecules}

The presumption that some substances can be extracted from the oxidized PE film by an aqueous medium and subsequently consumed by bacteria was tested with the help of

Table 3

ADP/ATP ratio for $N$. asteroides cultures at the end of the observation period

\begin{tabular}{lll}
\hline Substrate & Incubation (days) & ADP/ATP \\
\hline Oxidized HDPE & 120 & $2.26(0.09)$ \\
Oxidized LDPE & 120 & $1.75(0.19)$ \\
HDPE extract (control) & 120 & $3.51(0.30)$ \\
\hline
\end{tabular}

Standard deviations of the values are in brackets, $n \geq 2$. 
NMR spectroscopy. The aqueous extract from oxidized HDPE was prepared by the procedure described in the preceding section. ${ }^{1} \mathrm{H}$ NMR spectra showed the presence of significant amounts of organic substances (Fig. 4). On the whole the same organic species were observed in oxidized HDPE and LDPE extracts with chemical shifts ranging between 0.8 and 3.7 ppm (Fig. 4a and b). These chemical shifts are compatible with substituted or unsubstituted $\mathrm{CH}$ and $\mathrm{CH}_{2}$ groups, showing that these compounds are derivatives of short oxidized PE fragments but possibly also of the additive component (e.g. stearate). More precisely the two very well defined signals at $\delta=3.67$ and $1.20 \mathrm{ppm}$ correspond to ethanol (denoted 1). The presence of formate can also be noticed on both spectra resonating at $\delta=8.46 \mathrm{ppm}$ (not shown). Ethanol and formate are oxidation end-products of polyethylene. Although the extracted compounds are the same from the two types of polymers, two main differences can be observed: (i) integration of the different signals relative to that of the internal reference $\left(\mathrm{Tspd}_{4}\right)$ clearly showed that the amount of organic compounds extracted from LDPE (Fig. 4a) was $50 \%$ lower than that of HDPE (Fig. 4b), this

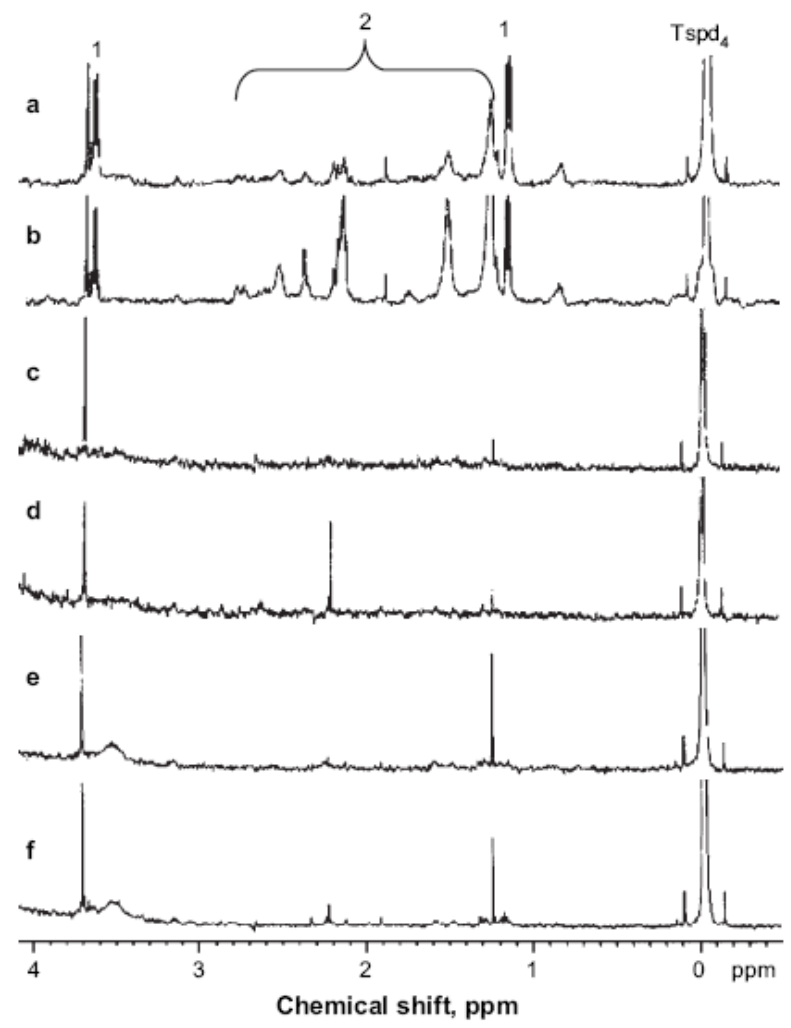

Fig. 4. ${ }^{1} \mathrm{H}$ NMR spectra of the oxidized HDPE and LDPE water phase extractable fractions and their consumption after incubation with $R$. rhodochrous. (a) Oxidized LDPE extractable fraction; (b) oxidized HDPE extractable fraction; (c) oxidized HDPE extractable fraction after 4 days incubation with $R$. rhodochrous; (d) oxidized LDPE extractable fraction after 4 days incubation with $R$. rhodochrous; (e) non-oxidized HDPE extractable fraction; (f) non-oxidized HDPE extractable fraction after 4 days incubation with $R$. rhodochrous, 1 : ethanol, 2: unknown organic compounds extracted from the polymers. is consistent with the ATP titration results and explicable by the thickness difference between HDPE and LDPE samples; (ii) the concentrations of ethanol and formate are rather constant in both samples while the other compounds (denoted 2) are in much higher amounts in HPDE sample. Fig. $4 c$ and d clearly demonstrates complete assimilation of the extracted species from both polymers after 4 days of incubation with $R$. rhodochrous. These results are consistent with the low ADP/ATP ratio measured after 4 days of incubation; the rapid initial growth of microorganisms was favoured by these easily metabolisable compounds extracted from the polymers. Also they showed that in the later phases of incubation no substrate was present in the cultures grown on extract from oxidized HDPE films which were used as references for the experiment with ATP determination (see Section 3.2.1) thus there was virtually no difference between this type of reference and an eventual reference consisting of pre-cultivated microbial cells in medium without substrate.

Finally Fig. 4e shows that no organic compounds are extracted from non-oxidized HDPE, so no leaching of the additives occurs in the absence of light or heat exposure. This result is consistent with the efficiency of abiotic treatment of PE in water (see Section 3.1) that indirectly proved this absence of leaching. As expected, no change in NMR spectrum was observed when non-oxidized HDPE was incubated with the Rhodococcus strain (Fig. 4f).

\subsection{Biofilm formation}

The surfaces of the film were observed by optical microscopy and scanning electron microscopy (SEM).

Because the tested PE films were transparent it was possible to use also transmission optical microscopy to observe the microorganisms on their surface. Application of this classical technique for the plastic film biodegradation study was quite original and brought the advantage of the fast and immediate monitoring of the film surface. More detailed microimages were obtained with SEM at the end of the incubation period.

Some of the tested microorganisms demonstrated their capacity to form biofilm on the surface of the oxidized LDPE and HDPE films tested. Bacteria R. rhodochrous and N. asteroides and fungus $M$. alpina were the most active (Figs. 5 and 6). Especially $N$. asteroides formed very dense filamentous mycelium on the surface. The biofilm development was the most progressive during the first 30 days of incubation. After this period no marked changes were identified by optical microscopy. Biofilm on the surface of the oxidized LDPE film was more dense and compact whereas on the oxidized HDPE film rather only islands of cells could be found indicating that probably important part of the population persists in the suspension form in the medium.

Contrary to previous studies $[3,4]$ no clear signs of bioerosion could be found on the surface of microorganism exposed films. However, it must also be noted that the attempts to wash the biofilm out of the material [4] before the SEM sample 

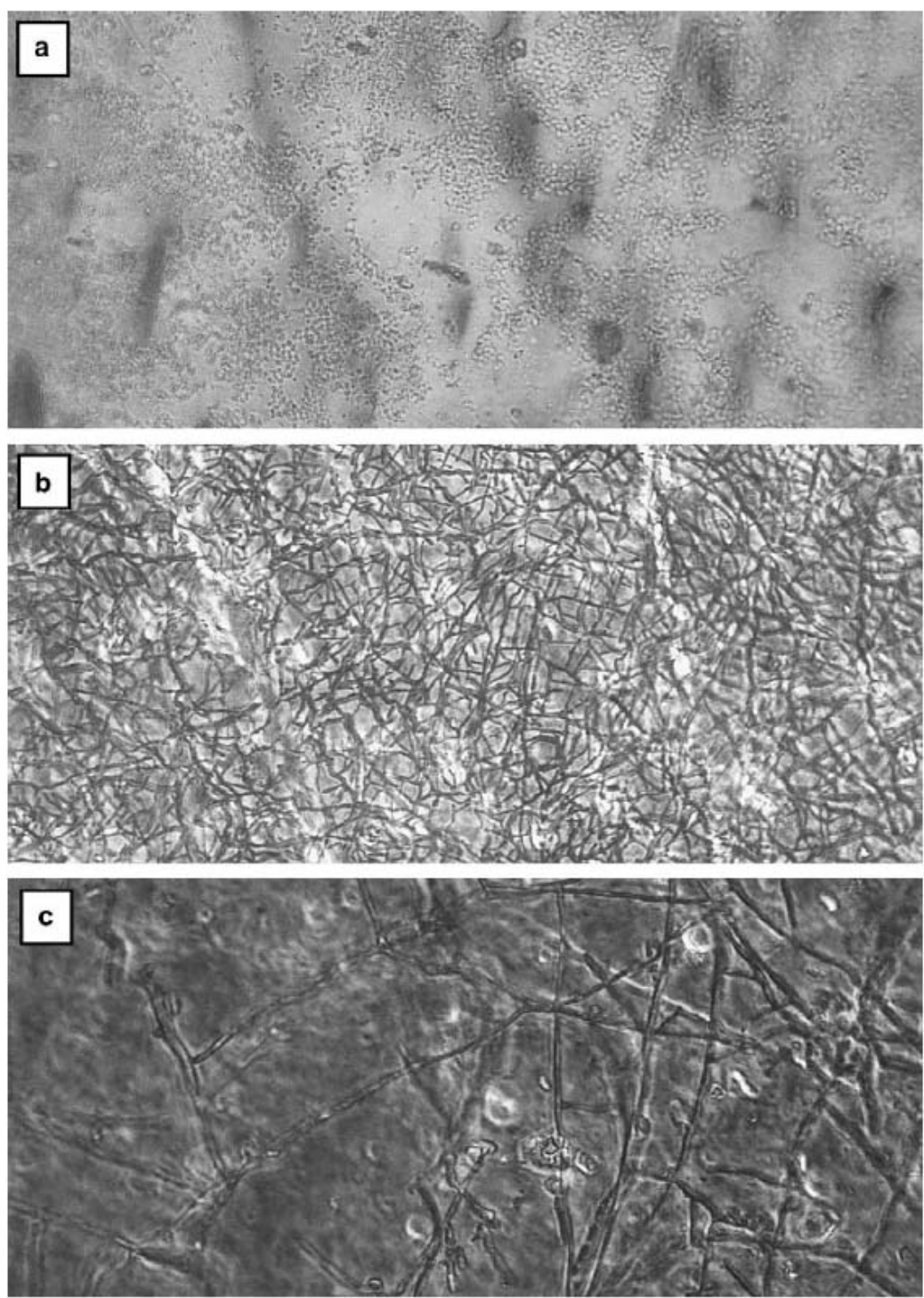

Fig. 5. Optical microscopy observation of the microorganisms on the oxidized LDPE film surface. Magnification cca. 100×; (a) R. rhodochrous (95 days of incubation); (b) N. asteroides ( 88 days of incubation); (c) M. alpina (70 days of incubation).

preparation were quite unsuccessful and that some bioerosion traces could stay hidden under the biofilm.

Existence of the biofilm was independently proved by $\mu$ ATR-FTIR spectroscopy in the cases of $R$. rhodochrous and $N$. asteroides on the oxidized LDPE film (Fig. 7). The bands at $1653 \mathrm{~cm}^{-1}$ and the nearby bands on its right can be assigned to protein material, the broad bands peaking at 1133 and $993 \mathrm{~cm}^{-1}$ showed the presence of polysaccharides.

\subsection{SEC analysis}

To test if the PE film degradation occurs in a significant part of the polymer volume the molecular weight distributions were compared between the material samples incubated in the presence of microorganisms and the samples incubated abiotically under identical conditions. The longest incubation was 203 days in the case of the oxidized HDPE with $R$. rhodochrous and 141 days in the case of the oxidized LDPE with $R$. rhodochrous. With M. alpina, A. flavus, $C$. cladosporoides and $N$. asteroides the periods of incubation were from 114 to 133 days. For both of the two materials the analysis did not reveal any significant difference in $M_{\mathrm{w}}$ distribution between the biotic sample and the abiotic control (data not shown). The observations suggest that the microbial attack was only superficial probably involving chain end carboxylic acids and that the microorganisms during the experiment period were not able to perturb the whole material volume. 

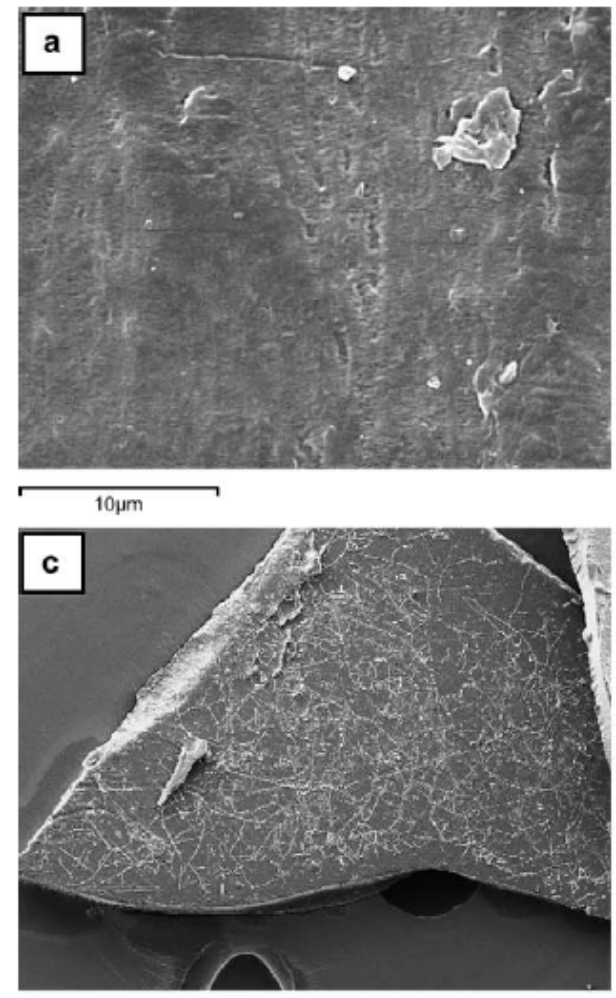

$500 \mu \mathrm{m}$

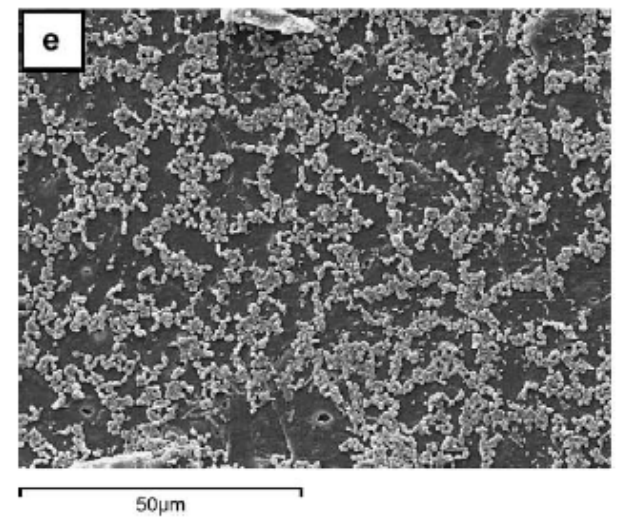

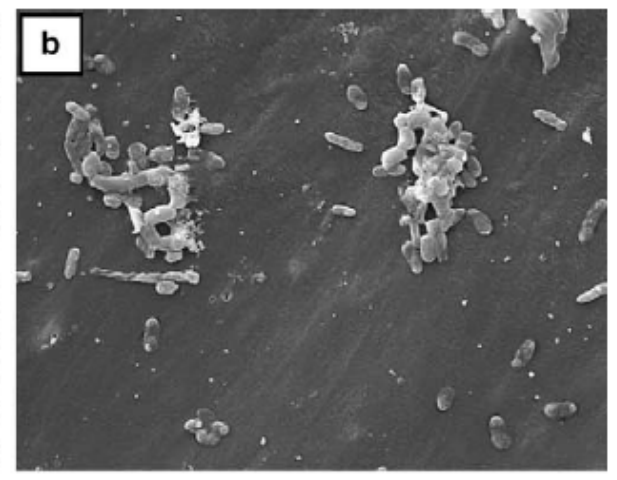
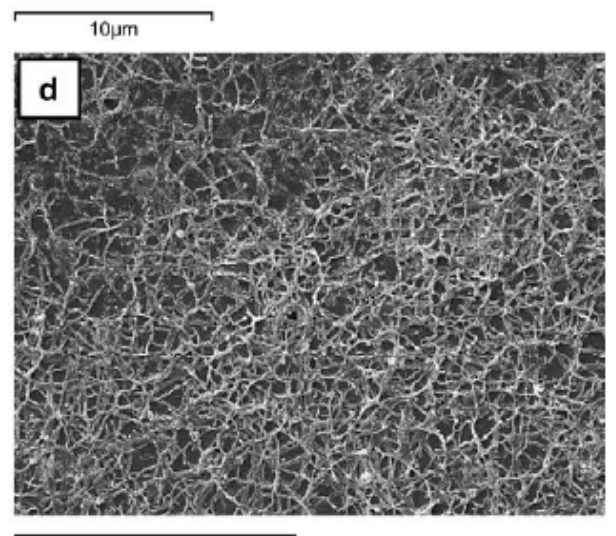

50 um

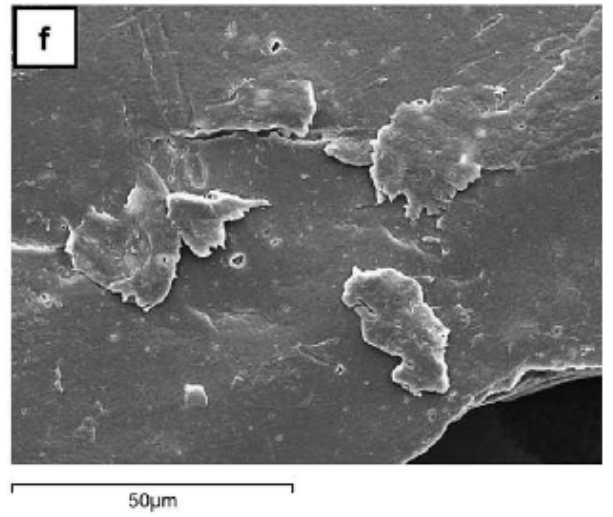

Fig. 6. SEM images of the tested materials after approximately 100 days of incubation with the designed microorganisms and the control samples incubated without microorganism (abiotic). (a) Oxidized HDPE abiotic; (b) oxidized HDPE $+R$. rhodochrous; (c) oxidized LDPE $+M$. alpina; (d) oxidized LDPE + N. asteroides; (e) oxidized LDPE + R. rhodochrous; (f) oxidized LDPE abiotic.

\section{Discussion}

The tested PE films represent a new branch of polymer materials with time programmed mechanical properties. The fine balance of antioxidant and pro-oxidant contents guarantees that after the preset period of service life relatively fast abiotic oxidation begins. As a consequence the material loses its mechanical properties and disintegrates into small fragments. These features provide a potential solution to the problems of "visual pollution" by plastic litter and its macro-toxicity in the environment that are constantly in the centre of public attention. On the molecular level the abiotic oxidation results in dramatic reduction in molecular weight, introduction of polar groups and increase in hydrophilicity. Evidently the oxidation renders the material much more vulnerable to microbial attack.

In accordance with other authors $[7,1]$ we observed that the early stage of biodegradation was characterized by fast growth probably mainly supported by the consumption of low molecular weight compounds extracted into the water phase. The compounds were probably the low molecular weight degradation product of polyethylene chains, most often terminated with carboxylic groups, already observed in other studies $[8,9]$. The fast initial phase was followed finally by 


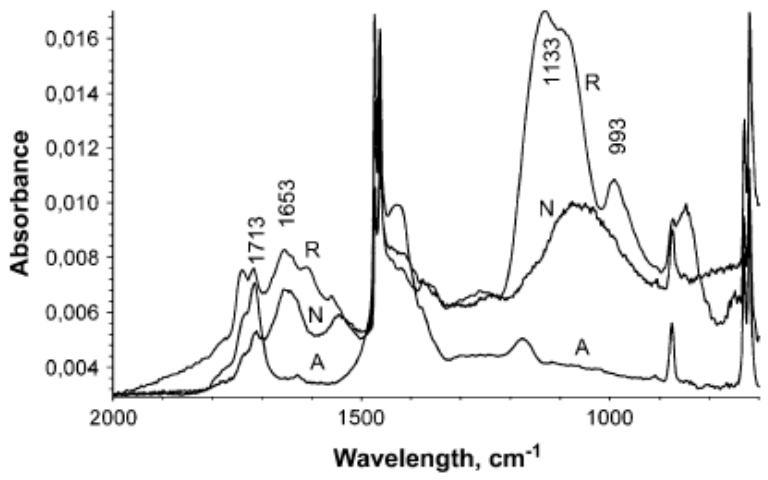

Fig. 7. ATR-FTIR spectra of the biofilm covered oxidized LDPE film. (A) Abiotic control; (N) N. asteroides culture (65 days of incubation); (R) R. rhodochrous culture ( 82 days of incubation).

stabilization of metabolic activity, which kept virtually the same level during many months. The data suggest that microorganisms continued to gain energy from the material hence the biodegradation was still going on even at some slow rate. However, it was not possible to make even a rough estimation of the process extent and eventually the time necessary for the ultimate biodegradation of the sample under applied laboratory conditions. It seems also that within the experiment period the observed microbial action was limited to the surface layer of the materials.

Some of the microbial strains tested in the study were able to form biofilm on the material surface, particularly $R$. rhodochrous and closely related $N$. asteroides. The same strains formed biofilm also on another pro-oxidant activated polyethylene film, as reported in a previous study [4]. Recently another Rhodococcus strain was isolated from soil and its capacity to form biofilm on similar type of material was demonstrated [10]. This group of bacteria, highly abundant in natural environments like soil, was identified as very active towards the higher molecular weight hydrocarbons [11]. One of the crucial features making them able to metabolise these water insoluble compounds could be the production of bio-surfactants [12] that render their surface more hydrophobic, so they can adhere to the hydrophilic surface of substrate, and in the same time mobilize substrate molecules and increase their accessibility for enzymes.

For the first time to our knowledge the adenine nucleotide determination was utilized for monitoring of material biodegradation. The method well known to biologists can produce interesting data about the energetic status of microorganisms, complementary to the classic methods in biodegradation studies.

\section{Acknowledgements}

The authors are grateful to Dr. Arne Lundin of Biothema for highly competent advice concerning luminometric methods and to Dr. Gerard Prensier for his help with optical microscopy. A.S. Martin is acknowledged for recording NMR spectra. The authors are also pleased to acknowledge the financial participation of EEC (Objective 3, European Social Fund); French government (DRIRE, Clermont-Ferrand, France); Conseil regional Auvergne, Clermont-Ferrand, France; and CCI Le Puy Yssingeaux, Haute Loire, France.

\section{References}

[1] Chiellini E, Corti A, Swift G. Polym Degrad Stab 2003;81:341-51.

[2] Jakubowicz I. Polym Degrad Stab 2003;80:39-43.

[3] Amaud R, Dabin P, Lemaire J, Al-Malaika S, Chohan S, Coker M, et al. Polym Degrad Stab 1994;46:211-24.

[4] Bonhomme S, Cuer A, Delort A-M, Lemaire J, Sancelme M, Scott G. Polym Degrad Stab 2003;81:441-52.

[5] Fanton E, Gazel A, Lemaire J. Revue Generale des Caoutchoucs et des. Plastiques 1984;641:75.

[6] Lundin A, Hasenson M, Persson J, Pousette A. Methods Enzymol $1986 ; 133: 27-42$.

[7] Hadad D, Geresh S, Sivan A. J Appl Microbiol 2005;98:1093-100.

[8] Albertsson A-C, Barenstedt C, Karlsson S. Acta Polym 1993;45:97-103.

[9] Albertsson A-C, Barenstedt C, Karlsson S, Lindberg T. Polymer 1995; 36:3075-83.

[10] Orr IG, Hadar Y, Sivan A. Appl Microbiol Biotechnol 2004;65:97-104.

[11] Larkin MJ, Kulakov LA, Allen C. Curr Opin Biotechnol 2005;16: $282-90$.

[12] Lang S, Philp JC. Antonie Van Leeuwenhoek 1998;74:59-70. 